\title{
Impact of preoperative education on pain outcomes after coronary artery bypass graft surgery
}

\author{
Judy Watt-Watson ${ }^{\mathrm{a}, *}$, Bonnie Stevens $\mathrm{s}^{\mathrm{b}, \mathrm{c}}$, Joel Katz ${ }^{\mathrm{d}, \mathrm{e}, \mathrm{f}}$, Judy Costello ${ }^{\mathrm{g}}$, \\ Graham J. Reid ${ }^{\text {h,i }}$, Tirone David ${ }^{\mathrm{j}}$ \\ ${ }^{\mathrm{a}}$ Faculty of Nursing and Centre for the Study of Pain, University of Toronto, $50 \mathrm{St}$ George Street, Toronto, Ont., Canada M5S $3 \mathrm{H} 4$ \\ ${ }^{\mathrm{b}}$ Faculties of Nursing and Medicine, University of Toronto, Toronto, Ont., Canada \\ ${ }^{\mathrm{c}}$ Hospital for Sick Children, Toronto, Ont., Canada \\ ${ }^{\mathrm{d}}$ Department of Psychology and School of Kinesiology and Health Science, York University, Toronto, Ont., Canada \\ ${ }^{\mathrm{e}}$ Acute Pain Research Unit, Department of Anesthesia, Toronto General Hospital and Mount Sinai Hospital, Toronto, Ont., Canada \\ ${ }^{\mathrm{f}}$ Departments of Anesthesia and Public Health Sciences, University of Toronto, Toronto, Ont., Canada \\ ${ }^{\mathrm{g}}$ Administration, Toronto General Hospital, Toronto, Ont., Canada \\ ${ }^{\mathrm{h}}$ Psychology and Family Medicine, University of Western Ontario, London, Ont., Canada \\ ${ }^{\mathrm{i}}$ Public Health Science and Medicine, University of Toronto, Toronto, Ont., Canada \\ ${ }^{j}$ Department of Surgery, Toronto General Hospital, Toronto, Ont., Canada
}

\begin{abstract}
Cardiovascular diseases cause more disability and economic loss in industrialized nations than any other group of diseases. In previous work [Nurs Res 49 (2000a) 1], most coronary artery bypass graft patients (CABG, $N=225$ ) reported unrelieved pain and received inadequate analgesics. This study proposed to evaluate a preadmission education intervention to reduce pain and related activity interference after CABG surgery. Patients $(N=406)$ were randomly assigned to (a) standard care or (b) standard care + pain booklet group. Data were examined at the preadmission clinic and across days 1-5 after surgery. Outcomes were pain-related interference (BPI-I), pain (MPQ-SF), analgesics (chart), concerns about taking analgesics (BQ-SF), and satisfaction (American Pain Society-POQ). The impact of sex was explored related to primary and secondary outcomes. The intervention group did not have better overall pain management although they had some reduction in pain-related interference in activities $(t(355)=2.54, P<0.01)$ and fewer concerns about taking analgesics $(F(1,313)=2.7, P<0.05)$ on day 5 . Despite moderate 24 -h pain intensity across 5 days, patients in both groups received inadequate analgesics (i.e. 33\% prescribed dose). Women reported more pain and pain-related interference in activities than men. The booklet was rated as helpful, particularly by women. In conclusion, the intervention did not result in a clinically significant improvement in pain management outcomes. In future, an intervention that considers sex-specific needs and also involves educating the health professionals caring for these patients may influence these results.
\end{abstract}

Keywords: Postoperative pain; Coronary artery bypass graft; Randomized controlled trial; Education intervention; Sex

\section{Introduction}

Cardiovascular diseases cause more disability and economic loss in industrialized nations than any other group of diseases (Sullivan and Spertus, 2001). Annual

* Corresponding author. Tel.: +1-416-978-2850; fax: + 1-416-978-8222. E-mail address: j.watt.watson@utoronto.ca (J. Watt-Watson). costs, including surgery, have been estimated at $\$ 18$ billion in Canada and $\$ 56$ billion in the United States (American Heart Association, 2002; Goldman and Braunwald, 1998; Heart and Stroke Foundation, 2003). Patients in cardiovascular settings, including those after coronary artery bypass graft (CABG) surgery, have reported considerable unrelieved pain (Puntillo, 1990; Puntillo and Weiss, 1994; Watt-Watson et al., 2000a). Pain from 
surgery has been one of the main reasons for emergency department visits and/or readmission for these patients (Sabourin and Funk, 1999; Weber et al., 1996). As well, women have reported greater chest discomfort than men and more problems with physical activities, although the latter were not examined related to pain (Artinian and Duggan, 1995; Baranson et al., 2000; King et al., 1994).

In our previous study of $225 \mathrm{CABG}$ patients, the majority received inadequate analgesics despite experiencing considerable pain and related activity interference after surgery (Watt-Watson et al., 2000a). Most patients would not voluntarily ask the nurse for analgesics and received only $47 \%$ of their prescribed dose. The Canadian Pain Society (Watt-Watson et al., 1999) and the Joint Commission on Accreditation of Healthcare Organizations (JCAHO, 2003) have emphasized the importance of effective management of acute pain to meet current requirements for earlier patient mobilization, reduced hospital stays, and reduced costs.

Previous studies of CABG preoperative teaching included minimal or no pain-related content, and the timing and type of teaching received minimal empirical attention. Interestingly, postoperative CABG patients themselves $(N=300)$ identified pain expectations as one potential area for improvement in discharge information (Beggs et al., 1998). Overall, structured preadmission cardiac education, either through pamphlets or teaching sessions within 2 weeks of surgery, appears to be more effective than postadmission informal teaching just prior to surgery (Christopherson and Pfeiffer, 1980; Cupples, 1990; Rice et al., 1992). However, the general education intervention in these studies did not change patients' analgesic intake or pain ratings after CABG surgery (Anderson, 1987; Rice et al., 1992; Schindler et al., 1989).

The degree to which a preoperative pain education intervention would reduce pain-related interference with activities, increase analgesic intake, decrease pain, and reduce patient concerns about taking analgesics is unknown. Therefore, a pilot study $(N=45)$ was implemented to evaluate the feasibility and clinical value of providing a booklet, Pain Relief After Surgery, in addition to standard preoperative education for patients scheduled for CABG surgery (Watt-Watson et al., 2000b). The booklet group received $46 \%$ more analgesics, had fewer concerns about taking analgesics and less painrelated interference despite unrelieved pain. Therefore, the booklet was deemed to be acceptable and feasible and required no changes prior to the present randomized controlled trial (RCT). The purpose of this study was to examine the impact of preoperative pain education on patients' activities, pain, analgesic use and related concerns, and length of hospital stay after CABG surgery and to explore the influence of factors such as sex on these outcomes.

\section{Methods}

\subsection{Design}

An RCT was used with elective-patients who were attending the standard preadmission education session 2-7 days before their CABG surgery. Repeated measurements were conducted to determine the impact of the pain education intervention vs. standard education on the primary outcome of pain-related interference in activities and on the secondary outcomes of postoperative pain, analgesic use, concerns about taking analgesics, satisfaction, and length of stay. The primary outcome was chosen based on our pilot work, where patients had significantly reduced pain-related interference in activities despite no changes in pain ratings (Watt-Watson et al., 2000b). For the present study, it was hypothesized that patients receiving the education intervention that focused on communicating pain and the use of analgesics would have reduced pain-related activity interference, particularly related to general activity, walking, deep breathing and coughing. The impact of sex was explored in relation to primary and secondary outcomes. All consenting patients completed baseline measures in the preadmission clinic before being randomized using a computergenerated randomization table to a standard care control group or a treatment group.

Data were collected preoperatively in the preadmission clinic and postoperatively in a large cardiovascular surgical unit with 85 beds in a large Toronto university-affiliated teaching hospital. The target population consisted of elective patients who were undergoing their first uncomplicated CABG surgery, attending a standard preadmission education session, and able to understand, read, and speak English. Patients were excluded if they were having repeat CABG and/or valve surgery.

\subsection{Procedure}

Ethical approval was received from the University Office of Research Services and the participating hospital's Research Ethics Board. Prior to the start of the study, meetings were held with the nurse managers and staff to explain the study protocol and to clarify related concerns. Eligible patients attending the preadmission session were informed of the study by the cardiovascular nurse coordinator who asked patients for their permission to release their name to the research assistant (RA). All patients who agreed were given verbal and written explanations of the study, including their rights, safeguards to preserve anonymity, and the risks and benefits of participation. Patients consenting to participate completed all questionnaires for baseline information prior to randomization.

Following randomization, all patients received the hospital's standard cardiovascular education which 
included a booklet and a video. These two components contained general information about the surgery, postoperative care and recovery, and a half-page of pain management guidelines included as a result of the inadequate pain management findings from our previous study (Watt-Watson et al., 2000a). In addition, patients randomly assigned to the intervention group received the intervention booklet from one research nurse, who instructed them using a standardized approach, to read it before surgery and bring it to hospital. She briefly discussed salient points contained in the booklet and answered patient questions. To maintain blinding of the RA and health professional staff, all patients received a brown envelope containing a folder with a copy of the consent and a letter of appreciation for participating in the study. In addition, patients assigned to the intervention group received the pain education intervention booklet in a similar folder in their envelope.

\subsection{Intervention}

The pain education intervention consisted of a booklet, Pain Relief After Surgery, that the investigators developed from previous research (APS, 1995; Ward et al., 1993; Watt-Watson et al., 2000a) and that reflected the Canadian Pain Society's position statement on pain relief (Watt-Watson et al., 1999). The 8-page booklet outlines the importance of pain relief to moving and breathing in order to prevent complications, how and when to ask for help with pain, pain relief methods including analgesic medications and non-pharmacological approaches, and concerns some patients have about requesting help with pain (see Appendix A). A differentiation is made between medication for moderate to severe pain vs. for mild pain. The booklet's content emphasizes the individuality of pain responses and the importance of good pain relief for recovery. Numerical rating scales (NRS), included to describe pain intensity and relief, were similar to those used in the more general cardiovascular surgery education booklet administered to all study participants. Common concerns that prevent patients from asking for help and/or taking analgesics were addressed.

The booklet's face validity was assessed by pain experts in nursing, psychology, and medicine. It was pretested for readability and comprehension at the Grade 6 level. In the pilot study, most (81\%) CABG patients and families read the booklet and rated it as very helpful.

\subsection{Measures}

The primary outcome was pain-related interference in activities in the previous $24 \mathrm{~h}$. The secondary outcomes were pain, analgesics prescribed and administered, concerns about reporting pain and taking analgesics, length of hospital stay, and patient satisfaction.

\subsubsection{Primary outcome}

A modified version of the Interference Subscale of the Brief Pain Inventory (BPI-I) was used to measure interference with activities because of pain in the previous $24 \mathrm{~h}$ at the baseline preadmission clinic and on days 3 and 5 after surgery (Daut et al., 1983; Levin et al., 1985; Serlin et al., 1995). Two items from the original measure relating to work and enjoyment of life were not considered relevant in this context and were not administered. An additional item about coughing and deep breathing was included as suggested in the American Pain Society-Patient Outcome Questionnaire (American Pain Society Quality of Care Committee (APS), 1995). This modified subscale has internal consistency and validity, including $\alpha=0.82$ for a surgical sample (McNeill et al., 1998) and 0.71 for our pilot study (Watt-Watson et al., 2001). The BPI-I was administered on day 3 , when increased ambulation usually results in peak pain levels, and on day 5 close to the time when patients on average are usually discharged. A total interference score $(0-60)$ is calculated by taking the sum of the six NRS $(0-10)$, with the anchors being does not interfere (0) and completely interferes (10). The six items, which determine whether pain is severe enough to interfere with general activities, sleep, mood, walking, deep breathing and coughing, or relations with others can be scored individually.

\subsubsection{Secondary outcomes}

Pain was measured by the McGill Pain QuestionnaireShort Form, which includes the Pain Rating Index (PRI) of 15 descriptors that describe the sensory and affective dimensions of pain, and the Present Pain Intensity (PPI) for a global measure of pain (Melzack, 1987). The daily NRS $(0-10)$ measured pain on moving at the time of the interview (i.e. pain now at mid-day or early afternoon) and the worst pain on moving in the previous $24 \mathrm{~h}$. The NRS (0-10) with an unpleasantness anchor, which was also used, has been established as a valid and reliable affective label (Gracely et al., 1978). Three pain rating scores from the PRI were calculated from the sum of the following intensity rank values: (a) sensory PRI-S (0-33), (b) affective PRI-A $(0-12)$, and (c) a total PRI-T (0-45). Finally, the PPI was used as a global measure to describe most severe pain overall in the previous $24 \mathrm{~h}$, where each number was associated with the following words: no pain (0), mild (1), discomforting (2), distressing (3), horrible (4) and excruciating (5) pain.

Data about analgesics prescribed and administered in the previous $24 \mathrm{~h}$ were gathered from the patient's chart daily for days 1-5 after surgery. Analgesic doses were converted to standardized parenteral morphine equivalents (Hardman and Limbird, 2001). Concerns about seeking help and taking analgesics were examined with a subscale derived from the Barriers Questionnaire (BQ), a 27-item instrument that has internal consistency, test-retest reliability, and construct and content validity (Ward et al., 1993). This subset, 
the BQ-SF, has established internal consistency $(\alpha=0.72)$ and test-retest reliability ( $r=0.85$; APS, 1995). The $\alpha$ for our pilot study was 0.85 . The NRS are summed for a total subscale score. Patient satisfaction was measured by three NRS (0-10) from the American Pain Society-Patient Outcome Questionnaire (APS-POQ; APS, 1995). The NRS ask for ratings of general satisfaction about overall pain treatment and the responses of nurses and physicians to their reports of pain, and are summed for a total subscale score $(0-30)$. Despite evidence of validity from previous work (Ware et al., 1983; Ware and Hays, 1988), the meaning and relevance of patient satisfaction questions are not always clear. However, satisfaction was included in this study as it was hypothesized that with education, patients may be more critical of the care they received. Length of hospital stay was reported in days from admission to discharge as recorded in the patient's chart.

\subsubsection{Additional questions}

Three additional items were administered using a 0-10 point NRS that provided helpful information in previous work (see Fig. 1). At day 5, patients in the intervention group were asked to indicate how much of the booklet they had read and to explain why it was useful or not. Patients who chose not to read it were asked the reasons for their decision.

\subsection{Sample size}

Two independent samples were compared. Sample size calculations were based on estimated group means from pilot data of 17.5 and 13.1 for pain interference and a within group SD of 14.0. The test of equality of means was carried out at the 0.05 level of significance. A sample size of 166 per group gave a probability of 0.80 of rejecting the null hypothesis of equal means if the alternative holds with an $\alpha=0.05$. Attrition in previous studies accounted for 6-10\% of patients, and more involvement was being asked of patients in this study. Therefore, the final sample was increased by $20 \%$ to account for patient attrition. Although no data existed on which to determine a clinically significant change in pain interference with activities, a moderate effect size (20\%) was considered to be clinically significant in this study.

\subsection{Data analysis}

The intention-to-treat principle was maintained so that individuals randomized to the intervention group were included in this group even if they did not participate in the intervention (e.g. reading the booklet or completing the measures postoperatively). Protocol compliant analyses were also completed to determine whether the intervention worked when people adhered to the instructions. An $\alpha=0.05$ was the level of significance used for all analyses. Intervention and standard care group data were compared to assess the comparability of groups at baseline using chisquare analysis for discrete level data and analysis of variance (ANOVA) for continuous level data. Descriptive statistics (i.e. averages, SDs, proportions) were used to summarize outcome variable data at all time periods.

For the primary outcome, a mixed repeated measures (RM) ANCOVA was performed to determine the efficacy of the pain education intervention vs. standard education alone on pain interference after surgery in terms of group (i.e. between subjects main effect) and time (i.e. within subjects main effect). Patient's age and sex were used as covariates. For secondary outcomes, separate ANOVAs were performed for each of the following dependent variables: postoperative pain, analgesic use, concerns, satisfaction,

1. How often did you tell the nurse when you needed a pain medication without being asked first?

$\begin{array}{llllllllllll} & 0 & 1 & 2 & 3 & 4 & 5 & 6 & 7 & 8 & 9 & 10 \\ \text { Never } & & & & & & & & & & \end{array}$

2. How much has medication relieved your pain when you move?

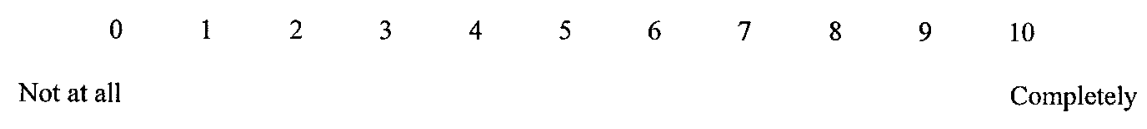

3. What pain did you usually have just before you received medication?

$\begin{array}{llllllllllll}0 & 1 & 2 & 3 & 4 & 5 & 6 & 7 & 8 & 9 & 10 \\ \text { No pain } & & & & & & & & & & & \\ & & & & & & & & & & & \\ \text { Worst pain } \\ \text { possible }\end{array}$

Fig. 1. Additional questions about pain and medication use in the previous $24 \mathrm{~h}$. 
and length of stay. A mixed between (treatment vs. standard care) and within (pre- vs. postintervention) ANOVA was performed for analgesic concerns to determine if the intervention had a significant impact in reducing patients' misbeliefs regarding analgesic use from baseline to day 5 after surgery. For significant ANOVAs with all outcomes, post hoc comparisons using Tukey's Honestly Significant Difference test were used to determine the source of the difference.

\section{Results}

The sample of 406 patients was accrued over 13 months from July 2000 to July 2001 The number of participants at each phase of the trial (i.e. enrolment, intervention allocation, follow-up, and analysis) is documented in the flow diagram in Fig. 2. No statistically significant differences between standard care and intervention groups were demonstrated at baseline in outcome variable data or patient characteristics. As the results of the intention to treat and protocol compliant analyses were the same, only the intention to treat analyses will be presented.

Patient characteristics and length of stay were similar for the standard care and intervention groups as outlined in Table 1. Of the 60 women, 26 were in the intervention group. Men, on average, were 61.9 years of age with 3.7 grafts, including $96 \%$ with an internal thoracic artery (ITA) graft. Women were older, with a mean age of 65.1 years and 3.2 grafts, including $92 \%$ with an ITA graft.

\subsection{Primary outcome}

\subsubsection{Pain-related interference with usual activities}

When an RM-ANCOVA was performed, there were no significant group by time interactions or main effects of group or time for overall pain-related interference with activities (day $3,16.55 \pm 11.88$ control vs. $16.06 \pm 12.35$; day $5, \quad 14.36 \pm 13.0$ control vs. $12.40 \pm 11.6$; $F(1,321)=0.48, P<0.49$ ). No effect of age or sex was demonstrated by study group. Sub-group analyses for treatment effects at specific days during recovery demonstrated that at day 5 , the standard care control group reported

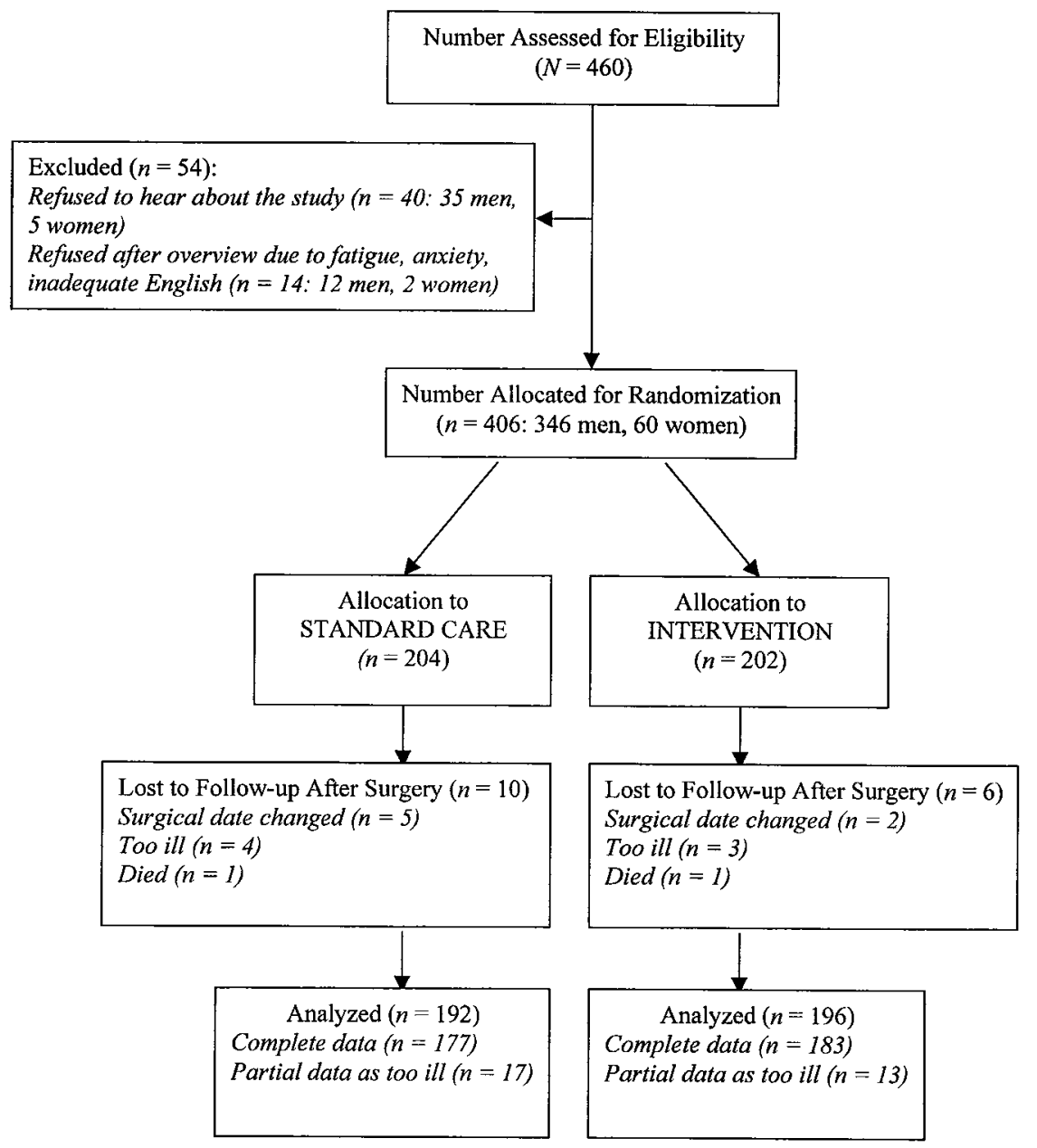

Fig. 2. Flow chart of the trial. 
Table 1

Characteristics and length of stay for the control and intervention groups

\begin{tabular}{lcc}
\hline Outcome & $\begin{array}{l}\text { Control }(M \pm \mathrm{SD}), \\
n=204\end{array}$ & $\begin{array}{l}\text { Intervention } \\
(M \pm \mathrm{SD}), n=202\end{array}$ \\
\hline $\begin{array}{l}\text { Characteristics } \\
\quad \text { Age (years) }\end{array}$ & $61.9 \pm 9.4$ & $61.7 \pm 9.3$ \\
$\quad$ Number of grafts & $3.4 \pm 0.9$ & $3.5 \pm 0.9$ \\
$\quad$ Postoperative & $5.9 \pm 2.7$ & $6.1 \pm 2.4$ \\
pain expected & & \\
by patients (0-10) & & $6.8 \pm 5.9$ \\
Length of stay (days) & $6.6 \pm 3.1$ & \\
\hline
\end{tabular}

${ }^{a}$ Differences using Student's $t$-tests not significant.

statistically significantly more pain-related interference than the intervention group in terms of overall scores $(15.5 \pm 13.8$ vs. $12.8 \pm 13.6 ; t(356)=2.01, P<0.01)$, and in particular, more interference related to deep breathing and coughing $(3.8 \pm 3.1$ vs. $2.7 \pm 3.1 ; t(355)=2.54$, $P<0.01$ ). For the total sample, it is noteworthy that at day $3,40 \%$ of patients reported moderate to severe overall interference because of pain $(\geq 4,0-10), 60 \%$ had moderate to severe interference due to pain with deep breathing and coughing, $41 \%$ with walking, $42 \%$ with general activity, $33 \%$ with mood, and $28 \%$ with sleep. At day 5, pain interference was still moderate to severe related to deep breathing and coughing (43\%), general activity $(33 \%), \operatorname{mood}(30 \%)$, sleep $(28 \%)$, and walking $(27 \%)$. Interference with relations with other people was minimal at both days 3 and 5 .

An RM-ANOVA was conducted to examine the primary outcome by sex for the total sample across days 3-5. Women had significantly greater interference in overall activities because of pain than men $(F(1,321)=4.29$, $P<0.04)$. Moreover, women's overall pain-related interference with activities remained the same or increased from day 3 to 5 , whereas men's scores decreased. In particular, women's scores were significantly different from men's for

Table 2

Pain-related interference in activities by sex on day 5 after surgery

\begin{tabular}{lccc}
\hline Outcome & Men $(M \pm \mathrm{SD})$ & Women $(M \pm \mathrm{SD})$ & $t$ (d.f. 356) \\
\hline $\begin{array}{l}\text { Interference } \\
\text { scores on day }\end{array}$ & & & \\
$\begin{array}{l}\text { 5 (BPI-I) } \\
\text { Total }\end{array}$ & $13.0 \pm 13.0$ & $20.1 \pm 16.2$ & $3.54^{* *}$ \\
Items & & & \\
$\quad$ General activity & $2.5 \pm 2.7$ & $4.3 \pm 3.5$ & $4.29^{* *}$ \\
$\quad$ Walking & $1.9 \pm 2.7$ & $3.6 \pm 3.3$ & $4.11^{* *}$ \\
$\quad \begin{array}{l}\text { Mood } \\
\text { Breathing and }\end{array}$ & $2.1 \pm 2.8$ & $3.4 \pm 3.5$ & $2.97^{* *}$ \\
coughing & $3.2 \pm 3.1$ & $4.1 \pm 3.3$ & $2.01^{*}$ \\
\hline
\end{tabular}

$* P<0.05 ; * * P<0.003$.

${ }^{a}$ Brief Pain Inventory-Interference. general activities $(F(1,316)=7.11, P<0.008)$ and walking $(F(1,305)=9.33, P<0.003)$. At day 5 , women had significantly more interference with overall activities in the total pain interference scores, and specifically with the BPI-I items of general activity, walking, mood, and deep breathing and coughing (see Table 2). The higher SDs associated with women's scores may reflect a lack of adequate power for this sub-group analysis.

\subsection{Secondary outcomes}

\subsubsection{Pain}

There were also no significant group by time interactions or main effects of group or time for any pain measure when an RM-ANCOVA was performed. No effect of age or sex was demonstrated by study group. For the total sample, the change in the mean (SD) for NRS for worst pain in the previous $24 \mathrm{~h}$ was statistically significant from day 2 $(6.23 \pm 2.74)$ to day $5(5.06 \pm 2.5 ; F(3,288)=4.63$, $P<0.3)$; however, it is of clinical significance that pain scores remained in the moderate range even at day 5 . NRS scores for the majority of patients were in the moderate to severe range from day $2(80 \%)$ to day 5 $(69 \%)$, including a considerable number in the severe range from day $2(50 \%)$ to day $5(30 \%$, see Table 3$)$. The majority of patients rated this pain as moderate to extremely unpleasant from day $2(71 \%)$ to day $5(65 \%)$, with $25 \%$ in the extremely unpleasant range at day 5 before discharge (see Table 3). A considerable number also had moderate to severe pain at the time of the interview (see Table 3). The mean (SD) levels of the PPI did not change significantly from day $2(2.41 \pm 1.28)$ to day $5(2.10 \pm 1.08)$. Similarly, the mean (SD) levels of

Table 3

Total sample percentage distribution scores in the moderate to severe range for pain and pain-related unpleasantness across 5 days after surgery

\begin{tabular}{lccccc}
\hline Measure (NRS ${ }^{\mathrm{a}}$ 0-10) & \multicolumn{3}{l}{ Day postsurgery } & & \\
\cline { 2 - 6 } & 1 & 2 & 3 & 4 & 5 \\
\hline Pain now (\%) & & & & & \\
Moderate (4-6) & 43 & 35 & 38 & 37 & 28 \\
Severe (7-10) & 34 & 28 & 18 & 11 & 10 \\
Total & 77 & 63 & 56 & 48 & 38 \\
Worst 24- $h$ pain (\%) & & & & & \\
Moderate (4-6) & 30 & 40 & 41 & 39 \\
Severe (7-10) & 50 & 43 & 35 & 30 \\
Total & 80 & 83 & 76 & 69 \\
Unpleasantness of $24-h$ pain $(\%)^{\mathrm{b}}$ & & & & \\
Moderate (4-6) & 28 & 38 & 46 & 40 \\
Extremely (7-10) & 43 & 37 & 22 & 25 \\
Total & 71 & 75 & 68 & 65 \\
\hline
\end{tabular}

${ }^{a}$ Numerical Rating Scales.

b Data collected from previous $24 \mathrm{~h}$ on days $2-5$ only. 
Table 4

Pain and unpleasantness ratings by sex across time

\begin{tabular}{lcl}
\hline Outcome & Men $(M \pm \mathrm{SD})$ & Women $(M \pm \mathrm{SD})$ \\
\hline Pain now on movement $(0-10)^{*}$ & \\
Day 1 & $5.2 \pm 2.4$ & $5.7 \pm 2.6$ \\
Day 2 & $4.7 \pm 2.7$ & $4.7 \pm 2.9$ \\
Day 3 & $3.8 \pm 2.3$ & $4.6 \pm 2.8$ \\
Day 4 & $3.5 \pm 2.3$ & $4.4 \pm 2.4$ \\
Day 5 & $3.0 \pm 2.2$ & $4.4 \pm 2.8$ \\
Worst pain in previous $24 h(0-10)^{* *}$ & \\
Day 2 & $6.1 \pm 2.7$ & $5.7 \pm 2.8$ \\
Day 3 & $5.8 \pm 2.5$ & $6.0 \pm 2.4$ \\
Day 4 & $5.4 \pm 2.6$ & $5.3 \pm 2.5$ \\
Day 5 & $4.9 \pm 2.5$ & $6.1 \pm 2.3$ \\
Unpleasantness of 24-h worst pain $(0-10)^{* *}$ & \\
Day 2 & $5.6 \pm 2.9$ & $5.3 \pm 2.9$ \\
Day 3 & $5.4 \pm 2.6$ & $5.4 \pm 2.8$ \\
Day 4 & $5.0 \pm 2.7$ & $4.7 \pm 2.6$ \\
Day 5 & $4.6 \pm 2.6$ & $5.9 \pm 2.5$ \\
Present Pain Intensity $(0-5)^{*}$ & \\
Day 2 & $2.5 \pm 1.3$ & $2.1 \pm 1.2$ \\
Day 3 & $2.4 \pm 1.1$ & $2.2 \pm 1.2$ \\
Day 4 & $2.1 \pm 1.1$ & $2.1 \pm 1.2$ \\
Day 5 & $2.1 \pm 1.1$ & $2.4 \pm 1.1$ \\
\hline
\end{tabular}

$* P<0.05 ; * * P<0.008$.

the PRI did not change significantly from day 2 $(9.63 \pm 8.19)$ to day $5(7.88 \pm 7.04)$, and scores were low. Most patients (80.3\%) expected to have moderate to severe pain after surgery. Pain around the chest incision area was the consistent site of the greatest pain across the week.

A significant effect of sex on pain scores was evident, using RM-ANOVA, for the total sample from day 2 to 5 after surgery (see Table 4). Women had greater pain on

Table 5

Daily analgesics administered and prescribed in milligrams morphine equivalents by study group across the five day study period

\begin{tabular}{lcc}
\hline Outcome & Control $(M \pm \mathrm{SD})$ & Intervention $(M \pm \mathrm{SD})$ \\
\hline Analgesic & administration (morphine equivalents & $m g / 24 h)^{\mathrm{a}}$ \\
Day 1 & $21 \pm 12$ & $21 \pm 11$ \\
Day 2 & $30 \pm 14$ & $29 \pm 15$ \\
Day 3 & $28 \pm 17$ & $27 \pm 16$ \\
Day 4 & $22 \pm 17$ & $22 \pm 15$ \\
Day 5 & $20 \pm 17$ & $20 \pm 16$ \\
Analgesic prescription (morphine equivalents & $m g / 24 h)^{\mathrm{a}}$ \\
Day 1 & $151 \pm 59$ & $152 \pm 59$ \\
Day 2 & $85 \pm 34$ & $85 \pm 35$ \\
Day 3 & $68 \pm 43$ & $62 \pm 27$ \\
Day 4 & $60 \pm 24$ & $57 \pm 16$ \\
Day 5 & $58 \pm 21$ & $56 \pm 14$ \\
\hline
\end{tabular}

a Differences not significant. movement at the time of the daily interview $(F(4,261)=4.87, P<0.03)$, more severe 24 -h pain $(F(3,288)=7.61, P<0.006)$, and greater overall pain (PPI; $F(3,278)=8.05, P<005)$ than did men. A signifcant sex effect also emerged for unpleasantness of the worst 24-h pain $(F(3,285)=7.11, P<0.008)$.

\subsubsection{Analgesics}

There were no significant group by time interactions or main effects of group or time for analgesics prescribed or administered. The analgesics prescribed were in the therapeutic range from day 1 , which included the intensive care period $(153 \pm 59 \mathrm{mg} / 24 \mathrm{~h})$, to day 5 (57 $\pm 17 \mathrm{mg} / 24 \mathrm{~h}$ morphine equivalents). However, the analgesics administered in the previous $24 \mathrm{~h}$ were inadequate for both study groups (see Table 5). Across all 5 days, the average 24-h dose for the total sample despite considerable pain on movement ranged from $20 \pm 12 \mathrm{mg}$ on day 1 to $20 \pm 16 \mathrm{mg} / 24 \mathrm{~h}$ on day 5 . On average, only $33 \%$ of the prescribed dose was given and usually when pain was at least 5 on the NRS $(0-10)$ for $53 \%$ of patients (see Fig. 1). Most patients stated that they never initiated a request for medication before they were asked whether analgesics were required $(1.60 \pm 2.0)$. However, the intervention group's negative response to this question was statistically significantly less ( 81 vs. $90 \%$ never asked, $P<0.01$ ). Most patients $(82 \%)$ had $80 \%$ relief or greater when analgesics were taken.

On day 4 , constipation was identified by $78 \%$ of the total group as the main side effect of the analgesics. Significantly more patients in the standard care group identified nausea as a side effect of analgesics than those in the intervention group (34 vs. $24 \%, P<0.000$ ).

\subsubsection{Concerns about asking for analgesics}

There were no group by time interactions or time or group main effects for concerns about asking for help with pain medication. However, the intervention group had statistically significantly fewer concerns about taking analgesics on day $5(22.6 \pm 14.72$ vs. $18.5 \pm 14.25$; $F(1,313)=2.7, P<0.05)$. As well, specific concerns about addiction were less for the intervention group than the standard care group at day $3(3.7 \pm 3.6$ vs. $4.8 \pm 3.8)$ and at day $5(3.2 \pm 3.3$ vs. $4.0 \pm 3.6 ; F(1,305)=2.06$, $P<0.04)$.

No sex or age effects were found for concerns about asking for help with medications from the preadmission clinic to day 5 . However, at day 3 , women had significantly more concerns overall than men about seeking help with medication for pain $(29.1 \pm 18.5$ vs. $22.4 \pm 16.2$; $t(312)=2.33, P<0.02)$.

\subsubsection{Length of stay}

The mean length of stay for the total group was $6.7 \pm 0.7$ days, with no significant differences between the standard 
care $(6.6 \pm 3.1)$ and the intervention $(6.8 \pm 5.9)$ groups. No effect of sex was demonstrated by the study group; however for the total sample, the women's hospital stay was 2 days longer on average than the men's $(8.81 \pm 10.05$ vs. $6.34 \pm 2.8 ; t(390)=3.78, P<0.000)$.

\subsubsection{Patient satisfaction and evaluation of the booklet}

There were no group by time interactions or time or group main effects for this outcome across days 3 and 5 . Patients were very satisfied with their overall care and both nurses' and doctors' responses to their reports of pain.

Twenty percent of the intervention group said they had not received the booklet. Of the remainder, $88 \%$ said they read all or most of the booklet and most evaluated it on day 5 as being very or extremely helpful $(68 \%)$ or helpful $(14 \%)$. The majority stated that their spouse read it as well $(74 \%)$. Although no data are available to explain why some did not rate the booklet as helpful, the men who read only $50 \%(n=10)$, or less than $25 \%(n=16)$, of the booklet said they were too busy, too tired, too anxious, or did not specify a reason. Most women (81\%) in the intervention group rated the booklet as extremely helpful. Only one woman did not read it, citing fatigue as the reason.

\section{Discussion}

No effect of the preoperative education intervention on pain management outcomes was evident for these CABG patients. While sub-group analyses for treatment effects at specific days during recovery demonstrated statistically significant differences in overall interference scores and deep breathing and coughing, they were not large enough to be clinically relevant. Similarly to previous studies (Anderson, 1987; Rice et al., 1992; Schindler et al., 1989), the intervention did not change patients' analgesic intake or pain ratings after surgery. It is noteworthy that a third of patients, mostly men, either did not remember receiving the booklet or did not read most of it. The timing of the intervention may be problematic, although based on research, and an additional telephone call to reinforce teaching will be considered in future. Greater quality monitoring of the intervention may have determined if the intervention nurse, who was not part of the surgical unit, appreciated the degree of patients' anxiety in her approach. As well, nurses were not included in an arm of this study to support the intervention because of contextual issues related to hospital mergers and layoffs.

Since hospital stays are now much shorter than previously, it is of special concern that a considerable number of the total sample had moderate to severe painrelated interference on both days 3 and 5, particularly related to deep breathing and coughing, general activity, walking, and mood. Patients continue to deal with these issues at home after discharge without health professional support (Beggs et al., 1998; Moore, 1994, 1995; Tack and Gillis, 1990; Wu, 1995), even up to 12 weeks postdischarge (Lenz and Perkins, 2000).--Several patients expressed concern about their pain management after discharge and hoped that "my doctor will give me pain killers when I go home". In several studies, patients identified the need for information about pain management to help with the recovery period after discharge (Beggs et al., 1998; Goodman, 1997). Weber et al. (1996) found that pain was one reason for emergency department visits associated with shortened hospital stays after CABG surgery. The present study underlines the potential problem of patients being discharged home with painrelated functional limitations that may predispose them to use expensive community services such as the emergency department.

Perhaps the most striking findings of the present study concerned the significant sex differences in pain interference and length of stay. The pattern of pain interference scores over time differed significantly for men and women. Women's pain-related interference scores were significantly higher than men's and increased from day 3 to 5 , whereas men's scores decreased over time. As well, the length of hospital stay was, on average, 2 days longer for women than for men. This study may not have been adequately powered for these sub-group analyses and these sex differences need to be examined further in future work. Shorter hospital stays and the increasing number of women undergoing $C A B G$ mean that these patients have greater immediate recovery needs. King (2000) found that women continued to report soreness in chest and leg incisions at 3 months after surgery. Moore and Dolansky (2001) emphasized the importance of facilitating women's home recovery after CABG because of their greater morbidity and mortality in the first year. This process is best initiated before discharge since women have fewer supports once they return home (Moore, 1995).

There was no impact of the intervention on the secondary outcomes of pain, analgesic use, concerns about taking analgesics, and length of hospital stay either by group or across time. Findings for the total sample indicated that the majority reported moderate to severe worst 24 -h pain on movement from day $2(80 \%)$ to day $5(69 \%)$, including a considerable number in the severe range from day $2(50 \%)$ to day $5(30 \%)$. Patients volunteered comments about pain including, "feels like a truck on my chest" and "pain is exhausting". Most patients rated this pain as moderate to extremely unpleasant from day $2(71 \%)$ to day $5(65 \%)$, with $25 \%$ in the extremely unpleasant range at day 5 before discharge. Unrelieved pain can precipitate a generalized sympathetic response, which involves the cardiovascular system (Cousins, 1994; Heye, 1991), and pre-existing 
coronary artery disease may exacerbate this problem. This consequence may be particularly true for women as there was a significant effect for pain measures across the postoperative period; women had significantly more pain than men and found it more unpleasant. In other research (Hawthorne, 1994; King, 2000), women have reported lower activity levels following CABG surgery and/or less relief of symptoms than men, including mediastinal incisional discomfort.

The analgesics administered in the previous $24 \mathrm{~h}$ were inadequate for both study groups, and there were no group or time effects by sex or age. Although analgesic use did not differ between groups, overall analgesic doses prescribed and administered for the total sample had improved when compared to previous data (Watt-Watson et al., 2000a). Nevertheless, on average, only $33 \%$ of the prescribed dose was given, despite the majority of patients' experiencing moderate to severe pain on day 5 just prior to discharge. Although concerns about seeking help with analgesics, including addiction, were statistically significantly different for the intervention group by day 5 , clinically this improvement in being less concerned about asking for assistance did not influence analgesics used or voluntary requests for analgesics. Women had more concerns than men about seeking help with medications for pain at day 3, including being concerned about addiction. Similar to findings in previous work, most patients identified constipation as the main side effect on day 4 and a reason for not taking analgesics.

In spite of the large sample size and statistical power, this study has several limitations. The severe fiscal constraints and substantial staffing changes were a major limitation. As a result, no concurrent pain education intervention was given to health professionals, although we had previously identified significant knowledge gaps in this setting (Watt-Watson et al., 2000b). As a result, the $20 \%$ of the intervention group who said they asked for help may not have received it. Moreover, $78 \%$ of patients on day 4 identified constipation as a reason for not taking analgesics, which reflects health professionals' lack of understanding of opioid management. Future research will include focus groups with staff in this setting to discuss their beliefs about why patients, especially women, are experiencing considerable pain and pain-related interference during their hospitalization, up to the day of discharge. Furthermore, the intervention in this study was provided almost exclusively by one research nurse. Although using a single person to deliver the intervention enhances a standardized delivery approach and minimizes individual variation, it could also introduce limitations related to the individual delivering the intervention. All patients came from one site, although it is the largest cardiovascular center in Canada.

Co-interventions such as pillows, positioning, shallow breathing, and/or immobility to decrease pain were not documented; however, they were available to all patients as part of standard care on the unit. In a randomized controlled trial, co-interventions may occur, but randomization would minimize the potential differences between the groups.

Although nurses were not informed of the nature of the intervention, unblinding may have occasionally occurred (e.g. they noticed the intervention booklet at the bedside or were asked about it by the patient). However, analgesic administration by nurses was low and pain ratings high, so that the likelihood that knowledge of the patient's group allocation influenced outcomes seems limited.

Patients in general have complained about health professionals' lack of adequate pain management following hospital discharge. It would be important to know the postdischarge course for these CABG patients. Data are currently being examined to determine the incidence of pain, analgesic use and side effects, complications such as infection, and the use of health care resources immediately following discharge.

In conclusion, the intervention did not result in overall better pain management, although there was some reduction in pain-related interference in activities and patient's concerns about taking analgesics by day 5. All patients received inadequate analgesics despite reporting moderate to severe pain during their 5-day hospital stay. Further exploration into why an interactive approach including the pain booklet did not work is required. Also, additional strategies besides the booklet are required to change ineffective pain practices; strategies especially need to include clinicians well versed in pain management. Sex differences were significant despite the proportionately smaller number of women in the sample. Almost all of the women read the booklet and evaluated it as extremely helpful, suggesting this intervention may be more appropriate for women. Although women had longer hospital stays, and more pain and related activity interference than did men up to the day of discharge, they were not given more analgesics than men. Strategies that focus on women's pain and related activity interference will be critical in reducing their significantly longer length of hospital stay as compared to men's. Although both men and women evaluated the intervention as helpful, sex-specific strategies that offer greater postoperative support and reinforcement need to be examined for their impact on improving patients' pain-related status before discharge.

\section{Acknowledgements}

The authors are indebted to all the patients who participated in this study. Funding was received from The Heart and Stroke Foundation of Ontario (Grant No. NA4174). 
Appendix A. Intervention booklet: pain relief after your surgery

This booklet discusses why pain relief is important, how and when to ask for help with pain, concerns patients have about seeking help with pain, and examples of pain relief methods.

\section{What is pain?}

Pain is an unpleasant feeling, such as soreness or discomfort, that is different for every person. Pain can tell you when something is wrong with your body and when you need to ask for help.

Surgery causes pain for most people because tissues are moved and/or cut. Pain that is not treated can be severe. It is important for your recovery not to have severe pain.

People feel pain differently, even if they have the same surgery. After surgery, nurses and doctors will ask you to rate your pain. They want you to tell them when you are hurting and how much the treatments relieve your pain. You are the only one who knows how your pain feels. Your help is very important to getting the best pain relief possible.

Why is pain relief so important?

People usually have some pain after surgery. Severe pain can prevent you from moving and breathing properly and can cause complications that make your hospital stay longer. It is important for you to have as little pain as possible to get well faster.

Good treatments are available to help relieve pain, especially medications after surgery. Other methods such as massage, deep breathing, and relaxation exercises also may help. Everyone's pain experience is different and you need to choose what works for you. You can use several methods at the same time.

People used to think that they had to "be strong" and "put up with severe pain." Now, doctors and nurses do not want you to do this because unrelieved pain can slow your recovery. Good pain relief is possible with your help.

\section{How and when do I ask for help with pain?}

It is very important for you to tell the nurses and doctors how much pain you are having, what it feels like, and whether treatments are working. If your pain rating is 4 or more, please tell the nurse.

1. A rating scale helps to tell us how much you hurt.

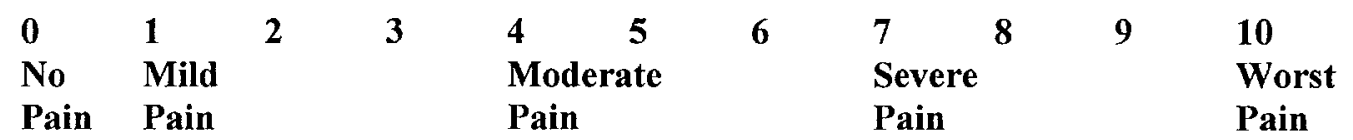

2. To ask for help, say to your nurse and/or doctor:

"My pain is 4 - I need something for pain"

How often should I ask for pain medication?

Medication usually relieves pain for about 4 hours. Therefore, to keep your pain rating below 4 when you are moving and/or doing your deep breathing exercises, you need to take your pain medication every 4 hours. You need less pain medication if you take it regularly than if you wait until your pain is severe. If you take the medication regularly and your pain is not below 4, ask for a stronger pill. 


\section{What drug treatments are available for pain relief?}

\section{A. Type:}

Strong medications to relieve moderate to severe pain can be taken by mouth such as morphine or Percocet $^{T M}$. Weaker medications such as Tylenol ${ }^{T M}$ and/or codeine help if pain is mild. If your medication in not working, please ask for a stronger one.

\section{B. Method:}

Your medication will first be given by intravenous (IV) into the vein. Some patients may be told by the nurse to use a special pump that gives you medication when you press a button.

Pills are used when you are allowed to drink. They can be as strong as IV or needles. If pain comes back before the next dose, ask for more pain relief.

All methods require that you take the medication regularly for pain relief

\section{What are patients' concerns?}

Many patients have concerns that stop them from telling someone about their pain and/or using pain medications. There are some responses to these concerns.

\section{Concern: $\quad \underline{\text { I am not a "good" patient if I tell someone about my pain. }}$}

\section{Response: $\quad 1 . " G o o d "$ patients DO tell when they hurt.}

You are a very important member of the pain management team and your help is needed. Please tell the nurse when you hurt and whether the pain treatment is working.

\section{Nurses EXPECT you to tell them when you hurt. They do not want you to} "handle it" by yourself.

You are helping by telling nurses when you hurt and if your medication is not working. They want you to have as little pain as possible. Tell them anything that has helped you with pain in the past.

\section{Pain does NOT mean you are healing.}

Unrelieved pain may slow healing and cause complications. People whose pain is wellcontrolled after surgery recover faster.

\section{Concern: I don't have pain, I have "discomfort" or "soreness".}

Response: $\quad$ Pain can be called other names. Use the pain scale to rate your word for pain such as "discomfort" or "soreness". If your rating is 4 or greater, discuss with your nurse about taking a pain medication. Not every person uses the word "pain".

Concern: $\quad \underline{\text { am afraid to take pain medication because of addiction and/or side effects. }}$

Response: $\quad 1$ Addiction is not a problem. It is rare $(<0.01 \%)$ for people taking medication for pain unless they already have a drug abuse problem. 
2.Constipation is preventable. Constipation can happen with pain medications. To prevent a problem, most patients need to take a stool softener and/or laxative while on medication. Talk to the nurse if you are not being given these.

3.Nausea is treatable. Do not refuse to take pain medication because of nausea. Nausea may happen when you first take pain pills. The doctor has ordered medication that usually takes the nausea away. Ask your nurse for this.

\title{
Concern: $\quad$ I don't want to have a needle.
}

Response: $\quad$ Strong pain medication does NOT have to be given by a needle.

Needles hurt and are not often used anymore. If you are allowed to drink, there are strong medications in pill form that you can take to help relieve your pain.

\section{What non-drug treatments are available for pain relief?}

Non-drug treatments can help to relieve your pain after surgery. Tell the nurse if there are methods that have helped you in the past. Patients have found the following helpful in addition to medications.

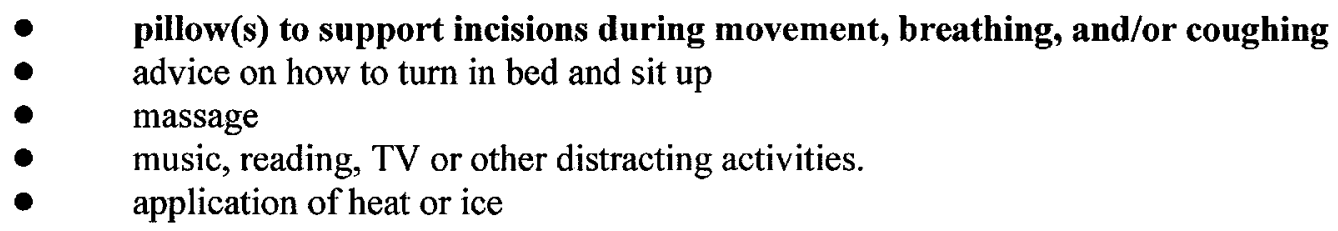

\author{
Remember \\ pain relief is important to your recovery \\ every person's pain is different \\ you are expected to tell us about your pain \\ good methods are available to control pain \\ YOU ARE A VERY IMPORTANT MEMBER OF THE PAIN MANAGEMENT TEAM
}

May be duplicated for use in clinical practice. From J. Watt-Watson, B. Stevens, J. Katz, J. Costello,

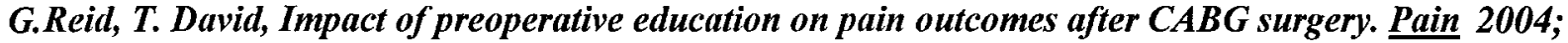
109: 73-85.

\section{References}

American Heart Association, Heart disease and stroke statistics-2003 update. Dallas, TX: American Heart Association; 2002

American Pain Society Quality of Care Committee (APS), Quality improvement guidelines for the treatment of acute and cancer pain. J Am Med Assoc 1995;274:1874-80.

Anderson E. Preoperative preparation for cardiac surgery facilitates recovery, reduces psychological distress, and reduces the incidence of acute postoperative hypertension. J Consult Clin Psychol 1987;55: 513-20.

Artinian NT, Duggan CH. Sex differences in patient recovery patterns after coronary artery bypass surgery. Heart Lung 1995;24:483-94.

Baranson S, Zimmerman L, Anderson A, Mohr-Burt S, Nieveen J. Functional status outcomes of patients with coronary artery bypass graft over time. Heart Lung 2000;29:33-46.

Beggs V, Willis S, Maislen E, Stoks T, White D, Sanford M, Becker A, Barber S, Pawlow P, Downs C. Patient education for discharge after coronary bypass surgery in the 1990s: are patients adequately prepared? J Cardiovase Nurs 1998;12:72-86.

Christopherson B, Pfeiffer C. Varying the timing of information to alter preoperative anxiety and postoperative recovery in cardiac surgery patients. Heart Lung 1980;9:854-61.

Cousins M. Acute and postoperative pain. In: Wall P, Melzack R, editors. Textbook of pain, 3rd ed. New York: Churchill Livingstone; 1994. p. $357-86$.

Cupples S. Effects of preadmission teaching on knowledge and postoperative recovery of coronary artery bypass graft patients. In: Funk $S$, Tornquist E, Champagne M, Copp L, Weise R, editors. Key aspects of recovery: improving nutrition, rest, and mobility. New York: Springer; 1990. p. 299-307.

Daut R, Cleeland C, Flanery R. Development of the Wisconsin Brief Pain Questionnaire to assess pain in cancer and other diseases. Pain 1983;17: 197-210.

Goldman L, Braunwald E, editors. Primary cardiology. New York: W.B. Saunders; 1998. p. 10. 
Goodman H. Patients' perceptions of their education needs in the first six weeks following discharge after cardiac surgery. J Adv Nurs 1997;25: 1241-51. . . . .

Gracely R, McGrath P, Dubner R. Ration scales of sensory and affective verbal pain descriptors. Pain 1978;5:5-18.

Hardman J, Limbird L, editors. Goodman and Gilman's pharmacological basis of therapeutics, 10th ed. New York: McGraw-Hill; 2001.

Hawthorne M. Sex differences in recovery after coronary bypass surgery. Image 1994;26:75-80.

Heart and Stroke Foundation. Statistics and background informationincidence of cardiovascular diseases; July 2003, http://www. heartandstroke.ca

Heye M. Pain and discomfort after coronary artery bypass surgery. Cardiovasc Nurs 1991;27:19-23.

Joint Commission on Accreditation of Healthcare Organizations (JCAHO). Comprehensive accreditation manual for hospitals: the official handbook; July 2003, http://www.jcaho.org

King KM. Sex and short-term recovery from cardiac surgery. Nurs Res 2000;49:29-36.

King KB, Porter LA, Rowe MA. Functional, social, and emotional outcomes in women and men in first year following coronary artery bypass surgery. J Womens Health 1994;3:347-54.

Lenz E, Perkins S. Coronary artery bypass graft surgery patients and their family member caregivers: outcomes of a family-focused staged psychoeducation program. Appl Nurs Res 2000;13:142-50.

Levin D, Cleeland C, Dar R. Public attitudes toward cancer pain. Cancer 1985;56:2337-9.

Melzack R. The short-form McGill Pain Questionnaire. Pain 1987;30: $191-7$.

Moore SM. Development of discharge information for recovery after coronary artery bypass surgery. Appl Nurs Res 1994;7:170-7.

Moore SM. A comparison of women and men's symptoms during home recovery after coronary artery bypass surgery. Heart Lung 1995;24: 495-501.

Moore SM, Dolansky MA. Randomized trial of a home recovery intervention following coronary artery bypass surgery. Res Nurs Health 2001;24:93-104.

McNeill J, Sherwood G, Starck P, Thompson C. Assessing clinical outcomes: patient satisfaction with pain management. J Pain Symptom Manage 1998;16:29-40.

Puntillo K. Pain experiences of intensive care unit patients. Heart Lung $1990 ; 19: 526-33$.
Puntillo K, Weiss S. Pain: its mediators and associated morbidity in critically ill cardiovascular surgical patients. Nurs Res 1994;43: $31-6$.

Rice V, Mullin M, Jarosz P. Preadmission self-instruction effects on postadmission and postoperative indicators in CABG patients: partial replication and extension. Res Nurs Health 1992;15:253-9.

Sabourin C, Funk M. Readmission of patients after coronary artery bypass graft surgery. Heart Lung 1999;28:243-50.

Schindler B, Shook L, Schwartz G. Beneficial effects of psychiatric intervention on recovery after coronary bypass graft surgery. Gen Hosp Psychiatry 1989;11:358-64.

Serlin R, Mendoza T, Nakamura Y, Edwards K, Cleeland C. When is cancer mild, moderate or severe? Grading pain severity by its interference with function. Pain 1995;61:277-84.

Sullivan M, Spertus J. Chest pain: cause and consequence of coronary artery bypass grafting. Pain 2001;92:1-3.

Tack BB, Gillis CL. Nurse-monitored cardiac recovery: a description of the first 8 weeks. Heart Lung 1990;19:491-9.

Ward S, Goldberg N, Miller-McCauley V, Mueller C, Nolan A, PawlikPlank D, Robbins A, Stormoen D, Weissman D. Patient-related barriers to management of cancer pain. Pain 1993;52:319-24.

Ware J, Hays R. Methods for measuring patient satisfaction with specific medical encounters. Med Care 1988;26:393-402.

Ware J, Snyder M, Wright W, Davies A. Defining and measuring patient satisfaction with care. Eval Prog Plan 1983;6:247-63.

Watt-Watson J, Clark J, Finley A, Watson P. Canadian Pain Society Position Statement on pain relief. Pain Res Manag 1999;4:75-8.

Watt-Watson J, Garfinkel P, Gallop R, Stevens B, Streiner D. The impact of nurses' empathic responses on patients' pain management in acute care. Nurs Res 2000a; 49:1-10.

Watt-Watson J, Stevens B, Costello J, Katz J, Reid G. Impact of preoperative education on pain management outcomes after surgery: a pilot. Can J Nurs Res 2000b;31:41-56.

Watt-Watson J, Stevens B, Streiner D, Garfinkel P, Gallop R, Relationship between pain knowledge and pain management outcomes for their postoperative cardiac patients. J Adv Nurs 2001;36:535-45.

Weber M, Dimengo M, Whitman GR, Roberts-Brown M, McNamara E, McCarthy P. Emergency department visits and hospital readmissions following cardiac surgery. Circulation 1996;94(Suppl):358.

$\mathrm{Wu}$ C. Assessment of postdischarge concerns of coronary artery bypass graft patients. J Cardiovasc Nurs 1995;10:1-7. 